\title{
Quality of Information Maximization in Two-Hop Wireless Networks
}

\author{
Sucha Supittayapornpong, Michael J. Neely \\ Department of Electrical Engineering \\ University of Southern California \\ Los Angeles, California \\ Email: supittay@usc.edu, mjneely@usc.edu
}

\begin{abstract}
An information collection problem in a wireless network with random events is considered. Wireless nodes report on each event using one of multiple reporting formats. Each format has a different quality and uses a different number of bits. Delivering all data in the highest quality format can overload system resources. The goal is to make intelligent format selection and routing decisions to maximize time-averaged information quality subject to network stability. Lyapunov optimization theory can be used to solve such a problem by repeatedly minimizing the linear terms of a quadratic drift-plus-penalty expression. To reduce delays, a novel extension of this technique that preserves the quadratic nature of the drift minimization while maintaining a separable decision structure is proposed. Also, paths are restricted to 1 or 2 hops to avoid high queuing delay. The resulting algorithm can push average information quality arbitrarily close to optimum, with a trade-off in average delay. The algorithm compares favorably to the basic drift-pluspenalty scheme in terms of backlog and delay.
\end{abstract}

\section{INTRODUCTION}

This paper investigates dynamic scheduling and data format selection in a network where multiple wireless devices, such as smart phones, report information to a receiver station. The devices together act as a pervasive pool of information about the network environment. Such scenarios have been recently considered, for example, in applications of social sensing [1] and personal environment monitoring [2], [3]. Sending all information in the highest quality format can quickly overload network resources. Thus, it is often more important to optimize the quality of information, as defined by the end-user, rather than the raw number of bits that are sent. The case for quality-aware consideration is made in [4], [5], [6]. Network management with quality of information awareness for wireless sensor networks is considered in [7]. More recently, quality metrics of accuracy and credibility are considered in [8], [9] using simplified models that do not consider the actual dynamics of a wireless network.

In this paper, we extend the quality-aware format selection problem in [9] to a dynamic network setting. We particularly focus on distributed algorithms for routing, scheduling, and format selection that jointly optimize quality of information. Specifically, we assume that random events occur over time

This material is supported in part by one or more of the following: the NSF Career grant CCF-0747525, the Network Science Collaborative Technology Alliance sponsored by the U.S. Army Research Laboratory W911NF-09-20053. in the network environment, and these can be sensed by one or more of the wireless devices, perhaps at different sensing qualities. At the transport layer, each device selects one of multiple reporting formats, such as a video clip at one of several resolution options, an audio clip, or a text message. Information quality depends on the selected format. For example, higher quality formats use messages with larger bit lengths. The resulting bits are handed to the network layer at each device and must be delivered to the receiver station over possibly time-varying channels. This delivery can be a direct transmission from a device to the receiver station via an uplink channel, or can take a 2-hop path that utilizes another device as relay (we restrict paths to at most 2-hops for tight control over network delays). An example is a single-cell wireless network with multiple smart phones and one base station, where each smart phone has $3 \mathrm{G}$ capabilities for uplink transmission and Wi-Fi capabilities for device-to-device relay transmission.

Such a problem can be cast as a stochastic network optimization and solved using Lyapunov optimization theory. A "standard" method is to minimize a linear term in a quadratic drift-plus-penalty expression [10], [11]. This can be shown to yield algorithms that converge to optimal average utility with a trade-off in average queue size. The linearization is useful for enabling decisions to be separated at each node. However, it can lead to larger queue sizes and delays. In this work, we propose a novel method that uses a quadratic minimization for the drift-plus-penalty expression, yet still allows separability of the decisions. This results in an algorithm that maintains distributed decisions across all nodes for format selection and routing, similar to the standard (linearized) drift-plus-penalty approach, but reduces overall queue size.

For the derived algorithm, each device observes its input queue length and then selects a format to report an event according to a simple rule. The routing decision for each group of bits is determined at each device by considering its input, uplink, and relay queues. Then, allocation of channel resources for direct transmission is determined from a base station after observing current uplink queues and channel conditions. For the relay transmission, an optimization problem involving relay queues and channel conditions are solved at the base station to determined an optimal transmission decision. This process can be decentralized if all channels are orthogonal.

Our analysis shows that the standard drift-plus-penalty 


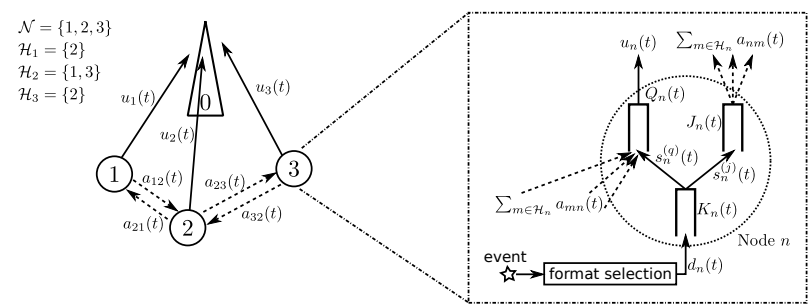

Fig. 1. An example network, illustrating the internal queues $K_{n}(t), Q_{n}(t)$, $J_{n}(t)$ for each node $n$.

algorithm and our new algorithm both converge to the optimal quality-of-information. The simulation also shows that the new algorithm has a significant savings in queue size and delay.

Thus, our contributions are twofold: (i) We formulate an important quality-of-information problem for reporting information in wireless systems. This problem is of recent interest and can be used in other contexts where "data deluge" issues require selectivity in reporting of information. (ii) We extend Lyapunov optimization theory by presenting a new algorithm that uses a quadratic minimization to reduce queue sizes while maintaining separability across decisions. This new technique is general and can be used to reduce queue sizes in other Lyapunov optimization problems.

In the next section we formulate the problem. Section III derives the novel quadratic algorithm. Section IV analyzes its performance. Section V presents simulation results.

\section{SYSTEM MODEL}

Consider a network with $N$ wireless devices, called nodes, that report information to a single base station. Let $\mathcal{N}=$ $\{1, \ldots, N\}$ be the set of wireless nodes, and denote the base station by node 0 . A network with $N=3$ nodes is shown in Fig. 1. The system operation has two stages, the format selection stage and the network routing and scheduling stage.

\section{A. Format Selection Stage}

Time is slotted to $t \in\{0,1,2, \ldots\}$. In every slot $t$, an event occurs with probability $\theta$, where $0<\theta \leq 1$. Each node $n \in \mathcal{N}$ on slot $t$ selects format $f_{n}(t)$ from a set of available formats $\mathcal{F}=\{0,1, \ldots, F\}$, where format selection affects quality and bit length for the reported information about the event. The quality can also depend on proximity of each node to the location of the event. To model this, the event on slot $t$ is described by a vector of event characteristics $\left\{\left(r_{n}^{(f)}(t), d_{n}^{(f)}(t)\right)\right\}$ for $n \in \mathcal{N}$ and $f \in \mathcal{F}$. Format $f \in \mathcal{F}$ for a node $n$ on slot $t$ corresponds to message length $d_{n}(t)=d_{n}^{(f)}(t)$ and quality $r_{n}(t)=r_{n}^{(f)}(t)$. The $\left(r_{n}^{(f)}(t), d_{n}^{(f)}(t)\right)$ values may be different on each slot. For example, they are identically $(0,0)$ for all formats at nodes $n$ that do not observe the current event (such as when the event is close to only a subset of the nodes), and are $(0,0)$ for all nodes and formats on slots in which no event occurs. To allow a node $n$ not to report on an event, there is a "blank format" $0 \in \mathcal{F}$ such that $d_{n}^{(0)}(t)=r_{n}^{(0)}(t)=0$ for all $t$. On each slot $t$, the vector $\left\{\left(r_{n}^{(f)}(t), d_{n}^{(f)}(t)\right)\right\}$ is assumed to be arbitrarily correlated over $n$ and $f$, but is independent and identically distributed (i.i.d.) across slots. Note that values $\left(r_{n}^{(f)}(t), d_{n}^{(f)}(t)\right)$ have arbitrary relationships, so $r_{n}^{\left(f_{1}\right)}(t)$ is not necessarily greater than $r_{n}^{\left(f_{2}\right)}(t)$ when $d_{n}^{\left(f_{1}\right)}(t)>d_{n}^{\left(f_{2}\right)}(t)$. Generally, it is possible to view $r_{n}^{(f)}(t)$ as the value or benefit of choosing format $f$. The i.i.d. assumption can be extended to a Markov model using techniques from [10], but we omit this for brevity. We assume that $0 \leq d_{n}(t) \leq d_{n}^{(\max )}$ and $0 \leq r_{n}(t) \leq r_{n}^{(\max )}$ for all $t$, for some positive real-valued constants $d_{n}^{(\max )}$ and $r_{n}^{(\max )}$.

\section{B. Network Routing and Scheduling}

At each node $n \in \mathcal{N}$, the $d_{n}(t)$ bits of data generated by format selection are put into input queue $K_{n}(t)$, as shown in Fig. 1. Each node has two orthogonal communication capabilities, called uplink transmission and ad-hoc relay transmission. The uplink transmission capability allows each node to communicate to the base station directly via an uplink channel. The relay capability allows communication between a node and its neighboring nodes. All transmissions are assumed to be successful by some feedback and forward error correction mechanisms. Choice of how many bits are used for each type of transmission is done at node $n$ by internally moving data from queue $K_{n}(t)$ to either uplink queиe $Q_{n}(t)$ or relay queиe $J_{n}(t)$. This is conceptually similar to the hop-count based queue architecture in [12].

In each slot $t$, let decision variables $s_{n}^{(q)}(t)$ and $s_{n}^{(j)}(t)$ represent the amount of bits in queue $K_{n}(t)$ that can be internally moved to $Q_{n}(t)$ and $J_{n}(t)$ (under the condition that enough bits are available in $K_{n}(t)$ ), as illustrated in Fig. 1. These bits can also be considered along with the remaining bits in the $Q_{n}(t)$ and $J_{n}(t)$ queues for uplink and relay transmission on slot $t$, since internal moving of bits is assumed to be done before transmissions start reading bits from the queues. Let $\left\{0,1, \ldots, s_{n}^{(q)(\max )}\right\}$ and $\left\{0,1, \ldots, s_{n}^{(j)(\max )}\right\}$ be the feasible ranges of $s_{n}^{(q)}(t)$ and $s_{n}^{(j)}(t)$, where constants $s_{n}^{(q)(\max )}$ and $s_{n}^{(j)(\max )}$ are assumed to be greater than or equal to the maximum number of bits that can be transmitted out of $Q_{n}(t)$ and $J_{n}(t)$, respectively, on one slot. Then the dynamics of input queue $K_{n}(t)$ are:

$$
K_{n}(t+1)=\max \left(K_{n}(t)-s_{n}^{(q)}(t)-s_{n}^{(j)}(t), 0\right)+d_{n}(t) .
$$

As a minor technical detail that is useful later, the $\max (\cdot, 0)$ operation above allows the $s_{n}^{(q)}(t)$ and $s_{n}^{(j)}(t)$ decisions to sum to more than $K_{n}(t)$. The actual $s_{n}^{(q)(\text { act })}(t)$ and $s_{n}^{(j)(\text { act) }}(t)$ bits moved from queue $K_{n}(t)$ thus satisfy:

$$
\begin{gathered}
s_{n}^{(q) \text { (act) }}(t)+s_{n}^{(j) \text { (act) }}(t)=\min \left(K_{n}(t), s_{n}^{(q)}(t)+s_{n}^{(j)}(t)\right) \\
0 \leq s_{n}^{(q)(\text { act })}(t) \leq s_{n}^{(q)}(t) \\
0 \leq s_{n}^{(j)(\text { act })}(t) \leq s_{n}^{(j)}(t)
\end{gathered}
$$

At node $n \in \mathcal{N}$, bits in relay queue $J_{n}(t)$ wait to be transmitted to one of node $n$ 's neighbors, which is denoted by set $\mathcal{H}_{n} \subseteq \mathcal{N}$. Node $n \in \mathcal{N}$ is capable of transmitting $a_{n m}(t)$ bits to node $m \in \mathcal{H}_{n}$ at time $t$, while this capability depends on relay channel condition $\gamma_{n m}(t)$ and the resource 
allocation policy. We assume that $0 \leq a_{n m}(t) \leq a_{n m}^{(\max )}$ for all $t$. Then the dynamic of queue $J_{n}(t)$ is

$$
J_{n}(t+1)=\max \left(J_{n}(t)-\sum_{m \in \mathcal{H}_{n}} a_{n m}(t)+s_{n}^{(j)(\mathrm{act})}(t), 0\right) .
$$

As before, the actual numbers of bits $a_{n m}^{(\text {act })}(t)$ for each node $n \in \mathcal{N}$ satisfy:

$$
\begin{gathered}
\sum_{m \in \mathcal{H}_{n}} a_{n m}^{\text {(act) }}(t)=\min \left(J_{n}(t)+s_{n}^{(j)(\text { act })}(t), \sum_{m \in \mathcal{H}_{n}} a_{n m}(t)\right) \\
0 \leq a_{n m}^{\text {(act) }}(t) \leq a_{n m}(t) \quad \text { for } m \in \mathcal{H}_{n} .
\end{gathered}
$$

We restrict all paths to at most 2 hops, and so all relayed bits that arrive to a node $n$ must be transmitted on the uplink channel at node $n$, and are thus put in the uplink queue $Q_{n}(t)$.

On each slot $t$, each node $n \in \mathcal{N}$ chooses to transmit $u_{n}(t)$ bits to the base station on its uplink channel. This decision depends on uplink channel condition $\gamma_{n 0}(t)$ and on the resource allocation policy. We assume that $0 \leq u_{n}(t) \leq u_{n}^{(\max )}$ for some positive real-valued $u_{n}^{(\max )}$. Then the dynamic of the uplink queue is:

$$
\begin{aligned}
Q_{n}(t+1)=\max \left(Q_{n}(t)-u_{n}(t)\right. & \left.+s_{n}^{(q)(\mathrm{act})}(t), 0\right) \\
& +\sum_{m \in \mathcal{H}_{n}} a_{m n}^{\text {(act) }}(t) .
\end{aligned}
$$

The queuing equations (5) and (8) involve the actual amounts of data, but they can be bounded using (3), (4) and (7) as

$$
\begin{gathered}
J_{n}(t+1) \leq \max \left(J_{n}(t)-\sum_{m \in \mathcal{H}_{n}} a_{n m}(t)+s_{n}^{(j)}(t), 0\right) \\
Q_{n}(t+1) \leq \max \left(Q_{n}(t)-u_{n}(t)+s_{n}^{(q)}(t), 0\right)+\sum_{m \in \mathcal{H}_{n}} a_{m n}(t) .
\end{gathered}
$$

The queue dynamics (1), (9), (10) do not require the actual variables $s_{n}^{(j) \text { (act) }}, s_{n}^{(q) \text { (act) }}(t), a_{n m}^{(\text {act })}(t)$, and are the only ones needed in the rest of the paper.

To simplify notation, let $\mathbf{a}_{n}(t)=\left(a_{n m}(t): m \in \mathcal{H}_{n}\right)$ be a vector of outgoing relay transmission decisions from node $n$, and $\mathbf{a}(t)=\left(\mathbf{a}_{n}(t): n \in \mathcal{N}\right)$. Also, let $\mathbf{u}(t)=$ $\left(u_{n}(t): n \in \mathcal{N}\right)$ be a vector of all uplink transmission decisions. Their channel conditions are denoted by $\gamma(t)=$ $\left(\gamma_{n m}(t): n \in \mathcal{N}, m \in \mathcal{H}_{n} \cup\{0\}\right)$. Then in each slot $t$, define the feasible sets of decision variables $\mathbf{a}(t)$ and $\mathbf{u}(t)$ by $\mathcal{A}_{\boldsymbol{\gamma}(t)}$ and $\mathcal{U}_{\gamma(t)}$ respectively. In the literature, these capabilities are generally presented by abstracting an underlying technology into the feasible sets [10], [13], [14]. Note that if all channels are orthogonal, the feasible sets of both transmission decisions are separable across links.

\section{Stochastic Network Optimization}

Here we define the problem of maximizing time-averaged quality of information subject to queue stability. We use the following definitions:

Definition 1: A queue $\{X(t): t \geq 0\}$ is strongly stable if

$$
\limsup _{t \rightarrow \infty} \frac{1}{t} \sum_{\tau=0}^{t-1} \mathbb{E}\{X(\tau)\}<\infty
$$

Definition 2: A network of queues is strongly stable if every queue in the network is strongly stable.
In words, definition 1 means that a queue is strongly stable if its average queue length is finite.

Let $y_{0}(t) \triangleq \sum_{n \in \mathcal{N}} r_{n}(t)$ be the total quality of information from format selection on slot $t$, and $y_{0}^{(\max )} \triangleq \sum_{n \in \mathcal{N}} r_{n}^{(\max )}$ is its upper bound. The time average total information quality is

$$
\bar{y}_{0} \triangleq \liminf _{t \rightarrow \infty} \frac{1}{t} \sum_{\tau=0}^{t-1} \mathbb{E}\left\{y_{0}(\tau)\right\} .
$$

It is our objective to solve:

$$
\begin{aligned}
\max & \bar{y}_{0} \\
\text { s.t. } & \text { Network is strongly stable. }
\end{aligned}
$$

This problem is always feasible because stability is trivially achieved if all nodes always select the blank format.

\section{Dynamic Algorithm}

This section derives a novel "quadratic policy" to solve problem (11). The policy gives faster convergence and smaller total queue backlog as compared to the "standard" drift-pluspenalty (or "max-weight") policy of [10], [11].

\section{A. Lyapunov Optimization}

In this system, define a quadratic Lyapunov function by $L(\Theta(t))=\frac{1}{2} \sum_{n \in \mathcal{N}}\left[K_{n}^{2}(t)+Q_{n}^{2}(t)+J_{n}^{2}(t)\right]$, where $\Theta(t)=\left(K_{n}(t), Q_{n}(t), J_{n}(t): n \in \mathcal{N}\right)$ represents all queues in the system. Then the Lyapunov drift, the difference of Lyapunov functions between two consecutive slots, is defined by $L(\Theta(t+1))-L(\Theta(t))$. Intuitively, this drift is used to show stability of a system. When queue lengths grow large beyond certain values, then the drift becomes negative and a system is stable because the negative drift roughly implies reduction of queue lengths.

In order to optimize $\bar{y}_{0}$ in (11), the drift-plus-penalty func$\operatorname{tion}^{1} L(\Theta(t+1))-L(\Theta(t))-V y_{0}(t)$ is considered, where $V \geq 0$ is a constant that determines a trade-off between queue backlog and proximity to optimal utility.

Lemma 1: Given $x, a, c \in \mathbb{R}_{+}$and $b \in \mathbb{R}$,

$$
\begin{aligned}
& {[\max (x-a+b, 0)+c]^{2}} \\
& \quad \leq \begin{cases}B_{+} \triangleq(x-a)^{2}+(x+b)^{2}+(x+c)^{2}-2 x^{2}+2 b c & , b \geq 0 \\
B_{-} \triangleq(x-a)^{2}+(x+b)^{2}+(x+c)^{2}-2 x^{2}-2 a b & , b \leq 0\end{cases}
\end{aligned}
$$

In addition, the upper bounds can be loosened to

$$
\begin{array}{ll}
B_{+} \leq x^{2}+2 x(-a+b+c)+a^{2}+(b+c)^{2} & , b \geq 0 \\
B_{-} \leq x^{2}+2 x(-a+b+c)+(a-b)^{2}+c^{2} & , b \leq 0
\end{array}
$$

Proof: For brevity, only the case with $b \geq 0$ is proved.

$$
\begin{aligned}
& {[\max (x-a+b, 0)+c]^{2}} \\
& \leq(x-a+b)^{2}+c^{2}+2 c \max (x-a+b, 0) \\
& \leq(x-a)^{2}+b^{2}+2 b(x-a)+c^{2}+2 c \max (x+b, 0) \\
& \leq(x-a)^{2}+(x+b)^{2}-x^{2}+c^{2}+2 c(x+b) \\
& =(x-a)^{2}+(x+b)^{2}+(x+c)^{2}-2 x^{2}+2 b c . \\
& \leq x^{2}+2 x(-a+b+c)+a^{2}+b^{2}+c^{2}+2 b c \\
& =x^{2}+2 x(-a+b+c)+a^{2}+(b+c)^{2} .
\end{aligned}
$$

\footnotetext{
${ }^{1}$ The minus sign in front of $V$ is because the quality of information can be viewed as a negative penalty.
} 
Inequalities (14) and (15) prove respectively relation (12) and (13) for $b \geq 0$.

Using queuing dynamic (1), (9), and (10), the drift-pluspenalty is upper bounded by (16) below. Then, using relation (12), the bound becomes (17) below:

$$
\begin{aligned}
L & (\Theta(\tau+1))-L(\Theta(\tau))-V y_{0}(\tau) \\
\leq & \frac{1}{2} \sum_{n \in \mathcal{N}}\left\{\left[\max \left(K_{n}(\tau)-s_{n}^{(q)}(\tau)-s_{n}^{(j)}(\tau), 0\right)+d_{n}(\tau)\right]^{2}-K_{n}(\tau)^{2}\right. \\
& +\left[\max \left(Q_{n}(\tau)-u_{n}(\tau)+s_{n}^{(q)}(\tau), 0\right)+\sum_{m \in \mathcal{H}(n)} a_{m n}(\tau)\right]^{2}-Q_{n}(\tau)^{2} \\
& \left.+\left[\max \left(J_{n}(\tau)-\sum_{m \in \mathcal{H}(n)} a_{n m}(\tau)+s_{n}^{(j)}(\tau), 0\right)\right]^{2}-J_{n}(\tau)^{2}-2 V r_{n}(\tau)\right\} \\
\leq & (16) \\
& =\sum_{n \in \mathcal{N}}\left\{\left[K_{n}(\tau)-s_{n}^{(q)}(\tau)\right]^{2}+\left[K_{n}(\tau)-s_{n}^{(j)}(\tau)\right]^{2}+\left[K_{n}(\tau)+d_{n}(\tau)\right]^{2}\right. \\
& +\left[Q_{n}(\tau)-u_{n}(\tau)\right]^{2}+\left[Q_{n}(\tau)+s_{n}^{(q)}(\tau)\right]^{2}+\left[Q_{n}(\tau)+\sum_{m \in \mathcal{H}_{n}} a_{m n}(\tau)\right]^{2} \\
& \left.+\left[J_{n}(\tau)-\sum_{m \in \mathcal{H}_{n}} a_{n m}(\tau)\right]^{2}+\left[J_{n}(\tau)+s_{n}^{(j)}(\tau)\right]^{2}-2 V r_{n}(\tau)+C_{n}(\tau)\right\}
\end{aligned}
$$

where

$$
\begin{aligned}
C_{n}(\tau) \triangleq-3 K_{n}(\tau)^{2}+2 s_{n}^{(q)}(\tau) s_{n}^{(j)}(\tau)-3 Q_{n}(\tau)^{2} & \\
& +2 s_{n}^{(q)}(\tau) \sum_{m \in \mathcal{H}_{n}} a_{m n}(\tau)-2 J_{n}(\tau)^{2} .
\end{aligned}
$$

Minimizing the actual drift-plus-penalty term (16) is computationally expensive. In this paper, we propose a novel quadratic policy, derived from (17), that preserves the quadratic nature of the actual minimization while keeping decisions separable. As a result, the policy leads to a separated control algorithm in Section III-B.

Definition 3: Every time slot $t$, the quadratic policy observes current queue backlogs $\Theta(t)$, random vectors $\left\{\left(r_{n}^{(f)}(t), d_{n}^{(f)}(t)\right)\right\}_{n \in \mathcal{N}, f \in \mathcal{F}}$ and $\gamma(t)$. Then it makes a decision according to the following minimization problem.

$$
\begin{array}{lll}
\min & \sum_{n \in \mathcal{N}}\left\{\left[K_{n}(t)-s_{n}^{(q)}(t)\right]^{2}+\left[K_{n}(t)-s_{n}^{(j)}(t)\right]^{2}+\left[K_{n}(t)+d_{n}(t)\right]^{2}\right. \\
& +\left[Q_{n}(t)-u_{n}(t)\right]^{2}+\left[Q_{n}(t)+s_{n}^{(q)}(t)\right]^{2}+\left[Q_{n}(t)+\sum_{m \in \mathcal{H}_{n}} a_{m n}(t)\right]^{2} \\
& \left.+\left[J_{n}(t)-\sum_{m \in \mathcal{H}_{n}} a_{n m}(t)\right]^{2}+\left[J_{n}(t)+s_{n}^{(j)}(t)\right]^{2}-2 V r_{n}(t)\right\} \\
\text { s.t. } & s_{n}^{(q)}(t) \in\left\{0,1,2, \ldots, s_{n}^{(q)(\max )}\right\}, s_{n}^{(j)}(t) \in\left\{0,1,2, \ldots, s_{n}^{(j)(\max )}\right\} \quad \forall n \in \mathcal{N} \\
& f_{n}(t) \in \mathcal{F}, d_{n}(t)=d_{n}^{\left(f_{n}(t)\right)}(t), r_{n}(t)=r_{n}^{\left(f f_{n}(t)\right)}(t) \quad \forall n \in \mathcal{N} \\
& \mathbf{a}(t) \in \mathcal{A}_{\gamma(t)}, \mathbf{u}(t) \in \mathcal{U}_{\gamma(t)}
\end{array}
$$

\section{B. Separability}

The control algorithm can be derived from the quadratic policy in Definition 3. The whole minimization can be done separately due to a unique structure of the quadratic policy. This leads to five subproblems, as described below.

At every slot $t$ each node $n \in \mathcal{N}$ observes input queue $K_{n}(t)$ and options $\left(r_{n}^{(f)}(t), d_{n}^{(f)}(t)\right)$ and chooses a format $f_{n}(t)$ according to the admission-control problem:

$$
\min _{f_{n}(t) \in \mathcal{F}}\left(K_{n}(t)+d_{n}^{(f(t))}(t)\right)^{2}-2 V r_{n}^{\left(f_{n}(t)\right)}(t)
$$

This is solved easily by comparing each option $f \in \mathcal{F}$.

Each node $n$ moves data from its input queue to uplink and relay queues according to the uplink routing problem

$$
\min _{s_{n}^{(q)}(t) \in\left\{0,1, \ldots, s_{n}^{(q)(\max )}\right\}}\left(K_{n}(t)-s_{n}^{(q)}(t)\right)^{2}+\left(Q_{n}(t)+s_{n}^{(q)}(t)\right)^{2}
$$

and relay routing problem

$$
\min _{s_{n}^{(j)}(t) \in\left\{0,1, \ldots, s_{n}^{(j)(\max )}\right\}}\left(K_{n}(t)-s_{n}^{(j)}(t)\right)^{2}+\left(J_{n}(t)+s_{n}^{(j)}(t)\right)^{2}
$$

These two subproblems can be solved in closed forms. Let $D_{Q}^{+}(t) \triangleq\left\lceil\frac{K_{n}(t)-Q_{n}(t)}{2}\right\rceil, D_{Q}^{-}(t) \triangleq\left\lfloor\frac{K_{n}(t)-Q_{n}(t)}{2}\right\rfloor$ and $g_{Q}(x, t)=\left(K_{n}(t)-x\right)^{2}+\left(Q_{n}(t)+x\right)^{2}$. Then choose

$s_{n}^{(q)}(t)=$

$\begin{cases}s_{n}^{(q)(\max )} & , K_{n}(t)-Q_{n}(t) \geq 2 s_{n}^{(q)(\max )} \\ \left.\operatorname{argmin}_{x \in\left\{D_{Q}^{+}(t), D_{Q}^{-}(t)\right.}\right\}^{g Q}(x, t) & , 0<K_{n}(t)-Q_{n}(t)<2 s_{n}^{(q)(\max )} \\ 0 & , K_{n}(t)-Q_{n}(t) \leq 0\end{cases}$

Also, let $D_{J}^{+}(t) \triangleq\left\lceil\frac{K_{n}(t)-J_{n}(t)}{2}\right], D_{J}^{-}(t) \triangleq\left\lfloor\frac{K_{n}(t)-J_{n}(t)}{2}\right\rfloor$ and $g_{J}(x, t)=\left(K_{n}(t)-x\right)^{2}+\left(J_{n}(t)+x\right)^{2}$. Then choose

$$
\begin{aligned}
& s_{n}^{(j)}(t)= \\
& \begin{cases}s_{n}^{(j)(\max )} & , K_{n}(t)-J_{n}(t) \geq 2 s_{n}^{(j)(\max )} \\
\arg \min _{x \in\left\{D_{J}^{+}(t), D_{J}^{-}(t)\right\}} g_{J}(x, t) & , 0<K_{n}(t)-J_{n}(t)<2 s_{n}^{(j)(\max )} \\
0 & , K_{n}(t)-J_{n}(t) \leq 0\end{cases}
\end{aligned}
$$

Note that the solutions from the quadratic policy are "smoother" as compared to the solutions from the max-weight policy that would choose "bang-bang" decisions of either 0 or $s_{n}^{(q)(\max )}$ for $s_{n}^{(q)}(t)$ (and 0 or $s_{n}^{(j)(\max )}$ for $s_{n}^{(j)}(t)$ ).

The uplink allocation problem is

$$
\min _{\mathbf{u}(t) \in \mathcal{U}_{\gamma(t)}} \sum_{n \in \mathcal{N}}\left(Q_{n}(t)-u_{n}(t)\right)^{2}
$$

and the relay allocation problem is

$$
\begin{aligned}
\min _{\mathbf{a}(t) \in \mathcal{A}_{\gamma(t)}} \sum_{n \in \mathcal{N}}( & \left.Q_{n}(t)+\sum_{m \in \mathcal{H}_{n}} a_{m n}(t)\right)^{2} \\
& +\left(J_{n}(t)-\sum_{m \in \mathcal{H}_{n}} a_{n m}(t)\right)^{2} .
\end{aligned}
$$

The sets $\mathcal{U}_{\gamma(t)}$ and $\mathcal{A}_{\gamma(t)}$ depend on a considered interference model and transmission technology. If these sets are convex, the corresponding problems are convex. If channels are orthogonal so the sets have a product form (as discussed in Section II-B), then the decisions are separable across nodes.

\section{Algorithm}

Each time slot $t$ :

- Each node $n \in \mathcal{N}$, knowing its $K_{n}(t), Q_{n}(t)$ and $J_{n}(t)$

- Observes $\left\{\left(r_{n}^{(f)}(t), d_{n}^{(f)}(t)\right)\right\}_{f \in \mathcal{F}}$ and solves (18) to obtain $f_{n}(t)$.

- Chooses $s_{n}^{(q)}(t), s_{n}^{(j)}(t)$ via (21), (22).

- Moves data from $K_{n}(t)$ to $Q_{n}(t)$ and $J_{n}(t)$ with $s_{n}^{(q)(\mathrm{act})}(t), s_{n}^{(j)(\mathrm{act})}(t)$ satisfying (2)-(4) and (6)-(7) with values of $s_{n}^{(q)}(t), s_{n}^{(j)}(t)$.

- Base station, with knowledge of $Q_{n}(t)$ and $J_{n}(t) n \in \mathcal{N}$

- Observes $\gamma(t)$

- Solves (23) to obtain $\mathbf{u}(t)$.

- Solves (24) to obtain $\mathbf{a}(t)$.

- Signals nodes $n \in \mathcal{N}$ to make transmissions.

After this process, queues $K_{n}(t+1), Q_{n}(t+1)$ and $J_{n}(t+1)$ are updated via (1), (5), (8). 


\section{Stability and Performance Bounds}

Compare the quadratic policy with any other policy. Let $\left(s_{n}^{(q)}(\tau), s_{n}^{(j)}(\tau), f_{n}(\tau): n \in \mathcal{N}\right), \mathbf{u}(\tau), \mathbf{a}(\tau)$ and $r_{n}(t) \triangleq r_{n}^{\left(f_{n}(t)\right)}(t), d_{n}(t) \triangleq d_{n}^{\left(f_{n}(t)\right)}(t)$ be the decision from the quadratic policy in Definition 3. Then, let $\left(\hat{s}_{n}^{(q)}(\tau), \hat{s}_{n}^{(j)}(\tau), \hat{f}_{n}(\tau): n \in \mathcal{N}\right), \hat{\mathbf{u}}(\tau), \hat{\mathbf{a}}(\tau)$ and $\hat{r}_{n}(t) \triangleq$ $r_{n}^{\left(\hat{f}_{n}(t)\right)}(t), \hat{d}_{n}(t) \triangleq d_{n}^{\left(\hat{f}_{n}(t)\right)}(t)$ be decision variables from any other policy. From (17) and definition 3, the drift-plus-penalty under quadratic policy is bounded by (25) and is further bounded by (26) under any other policy as

$$
\begin{aligned}
& L(\Theta(\tau+1))-L(\Theta(\tau))-V y_{0}(t)(\tau) \\
& \leq \frac{1}{2} \sum_{n \in \mathcal{N}}\left\{\left[K_{n}(\tau)-s_{n}^{(q)}(\tau)\right]^{2}+\left[K_{n}(\tau)-s_{n}^{(j)}(\tau)\right]^{2}+\left[K_{n}(\tau)+d_{n}(\tau)\right]^{2}\right. \\
& \quad+\left[Q_{n}(\tau)-u_{n}(\tau)\right]^{2}+\left[Q_{n}(\tau)+s_{n}^{(q)}(\tau)\right]^{2}+\left[Q_{n}(\tau)+\sum_{m \in \mathcal{H}_{n}} a_{m n}(\tau)\right]^{2} \\
& \left.\quad+\left[J_{n}(\tau)-\sum_{m \in \mathcal{H}_{n}} a_{n m}(\tau)\right]^{2}+\left[J_{n}(\tau)+s_{n}^{(j)}(\tau)\right]^{2}-2 V r_{n}(\tau)+C_{n}(\tau)\right\}
\end{aligned}
$$

$$
\begin{aligned}
\leq & \frac{1}{2} \sum_{n \in \mathcal{N}}\left\{\left[K_{n}(\tau)-\hat{s}_{n}^{(q)}(\tau)\right]^{2}+\left[K_{n}(\tau)-\hat{s}_{n}^{(j)}(\tau)\right]^{2}+\left[K_{n}(\tau)+\hat{d}_{n}(\tau)\right]^{2}\right. \\
& +\left[Q_{n}(\tau)-\hat{u}_{n}(\tau)\right]^{2}+\left[Q_{n}(\tau)+\hat{s}_{n}^{(q)}(\tau)\right]^{2}+\left[Q_{n}(\tau)+\sum_{m \in \mathcal{H}_{n}} \hat{a}_{m n}(\tau)\right]^{2} \\
& +\left[J_{n}(\tau)-\sum_{m \in \mathcal{H}_{n}} \hat{a}_{n m}(\tau)\right]^{2}+\left[J_{n}(\tau)+\hat{s}_{n}^{(j)}(\tau)\right]^{2}-2 V \hat{r}_{n}(\tau)+\hat{C}_{n}(\tau) \\
& \left.+C_{n}(\tau)-\hat{C}_{n}(\tau)\right\}
\end{aligned}
$$

where

$$
\begin{aligned}
\hat{C}_{n}(\tau) \triangleq-3 K_{n}(\tau)^{2}+2 \hat{s}_{n}^{(q)}(\tau) \hat{s}_{n}^{(j)}(\tau)-3 Q_{n}(\tau)^{2} & \\
& +2 \hat{s}_{n}^{(q)}(\tau) \sum_{m \in \mathcal{H}_{n}} \hat{a}_{m n}(\tau)-2 J_{n}(\tau)^{2}
\end{aligned}
$$

From the second set of bounds (13), it follows that

$$
\begin{aligned}
& L(\Theta(\tau+1))-L(\Theta(\tau))-V y_{0}(\tau) \\
& \leq \sum_{n \in \mathcal{N}}\left\{K_{n}(\tau)\left[\hat{d}_{n}(\tau)-\hat{s}_{n}^{(q)}(\tau)-\hat{s}_{n}^{(j)}(\tau)\right]\right. \\
& \quad+Q_{n}(\tau)\left[\hat{s}_{n}^{(q)}(\tau)+\sum_{m \in \mathcal{H}_{n}} \hat{a}_{m n}(\tau)-\hat{u}_{n}(\tau)\right] \\
& \quad+J_{n}(\tau)\left[\hat{s}_{n}^{(j)}(\tau)-\sum_{m \in \mathcal{H}_{n}} \hat{a}_{n m}(\tau)\right] \\
& \left.\quad-V \hat{r}_{n}(\tau)\right\}+A(\tau)
\end{aligned}
$$

where

$$
\begin{aligned}
A(\tau) \triangleq & \frac{1}{2} \sum_{n \in \mathcal{N}}\left\{C_{n}(\tau)-\hat{C}_{n}(\tau)+\left(\hat{s}_{n}^{(q)}(\tau)+\hat{s}_{n}^{(j)}(\tau)\right)^{2}+\hat{d}_{n}(\tau)^{2}+\hat{s}_{n}^{(j)}(\tau)^{2}\right. \\
& \left.+\hat{u}_{n}(\tau)^{2}+\left(\hat{s}_{n}^{(q)}(\tau)+\sum_{m \in \mathcal{H}_{n}} \hat{a}_{m n}(\tau)\right)^{2}+\left(\sum_{m \in \mathcal{H}_{n}} \hat{a}_{n m}(\tau)\right)^{2}\right\} \\
\leq A \triangleq & \frac{1}{2} \sum_{n \in \mathcal{N}}\left\{2 s_{n}^{(q)(\max ) 2}+2 s_{n}^{(j)(\max ) 2}+d_{n}^{(\max ) 2}+u_{n}^{(\max ) 2}\right. \\
& +\left(\sum_{m \in \mathcal{H}_{n}} a_{m n}^{(\max )}\right)^{2}+\left(\sum_{m \in \mathcal{H}_{n}} a_{n m}^{(\max )}\right)^{2} \\
& \left.+2 s_{n}^{(q)(\max )} s_{n}^{(j)(\max )}+2 s_{n}^{(q)(\max )} \sum_{m \in \mathcal{H}_{n}} a_{m n}^{(\max )}\right\}
\end{aligned}
$$

The derivations (25)-(27) show that applying the quadratic policy to the drift-plus-penalty expression leads to the bound (27) which is valid for every other control policy. However, this linear minimization does not resemble quadratic minimization of the actual drift-plus-penalty term (16). The effects of the two policies are revealed in section $\mathrm{V}$ where the quadratic policy leads to a smaller queue length.

To analyze the system, a conditional expectation of drift- plus-penalty is derived from (27) and (28) as

$$
\begin{aligned}
\mathbb{E} & \left\{L(\Theta(\tau+1))-L(\Theta(\tau))-V y_{0}(\tau) \mid \Theta(\tau)\right\} \\
\leq & \sum_{n \in \mathcal{N}}\left\{K_{n}(\tau) \mathbb{E}\left\{\hat{d}_{n}(\tau)-\hat{s}_{n}^{(q)}(\tau)-\hat{s}_{n}^{(j)}(\tau) \mid \Theta(\tau)\right\}\right. \\
& +Q_{n}(\tau) \mathbb{E}\left\{\hat{s}_{n}^{(q)}(\tau)+\sum_{m \in \mathcal{H}_{n}} \hat{a}_{m n}(\tau)-\hat{u}_{n}(\tau) \mid \Theta(\tau)\right\} \\
& +J_{n}(\tau) \mathbb{E}\left\{\hat{s}_{n}^{(j)}(\tau)-\sum_{m \in \mathcal{H}_{n}} \hat{a}_{n m}(\tau) \mid \Theta(\tau)\right\} \\
& \left.-V \mathbb{E}\left\{\hat{r}_{n}(\tau) \mid \Theta(\tau)\right\}\right\}+A .
\end{aligned}
$$

Let $\left.\omega(t)=\left(\gamma(t),\left\{\left(r_{n}^{(f)}(t), d_{n}^{(f)}(t)\right)\right\}_{n \in \mathcal{N}, f \in \mathcal{F}}\right\}\right)$ be a vector of every randomness in this system at time $t$. As discussed in Section II, $\omega(t)$ is i.i.d. over slots and is assumed to have distribution $\pi(\omega)$. Define an $\omega$-only policy as one that make a (possibly randomized) choice of decision variables based only on the observed $\omega(t)$. Then we customize an important theorem from [10].

Theorem 1: When problem (11) with stationary distribution $\pi(\omega)$ is feasible, then for any $\delta>0$ there exists an $\omega$-only policy that chooses all controlled variables $\left(f_{n}^{*}(t), s_{n}^{(q) *}(t), s_{n}^{(j) *}(t): n \in \mathcal{N}\right), \mathbf{u}^{*}(t), \mathbf{a}^{*}(t)$, and

$$
\begin{aligned}
\mathbb{E}\left\{y_{0}^{*}(t)\right\} & \leq y_{0}^{(\mathrm{opt})}+\delta & & \\
\mathbb{E}\left\{d_{n}^{*}(t)-s_{n}^{(q) *}(t)-s_{n}^{(j) *}(t)\right\} & \leq \delta & & n \in \mathcal{N} \\
\mathbb{E}\left\{s_{n}^{(q) *}(t)+\sum_{m \in \mathcal{H}_{n}} a_{m n}^{*}(t)-u_{n}^{*}(t)\right\} & \leq \delta & & n \in \mathcal{N} \\
\mathbb{E}\left\{s_{n}^{(j) *}(t)-\sum_{m \in \mathcal{H}_{n}} a_{n m}^{*}(t)\right\} & \leq \delta & & n \in \mathcal{N}
\end{aligned}
$$

where $y_{0}^{\text {(opt) }}$ is the optimal solution of problem (11). Also, $y_{0}^{*}(t) \triangleq \sum_{n \in \mathcal{N}} r_{n}^{*}(t)$ when $r_{n}^{*}(t) \triangleq r_{n}^{\left(f_{n}^{*}(t)\right)}(t)$ and $d_{n}^{*}(t) \triangleq$ $d_{n}^{\left(f_{n}^{*}(t)\right)}(t)$.

We additionally assume all constraints of the network can be achieved with $\epsilon$ slackness [10]:

Assumption 1: There are values $\epsilon>0$ and $0 \leq y_{0}^{(\epsilon)} \leq$ $y_{0}^{(\max )}$ and an $\omega$-only policy choosing all controlled variables $\left(f_{n}^{*}(t), s_{n}^{(q) *}(t), s_{n}^{(j) *}(t): n \in \mathcal{N}\right), \mathbf{u}^{*}(t), \mathbf{a}^{*}(t)$ that satisfies

$$
\begin{aligned}
& \mathbb{E}\left\{y_{0}^{*}(t)\right\}=y_{0}^{(\epsilon)} \\
& \mathbb{E}\left\{d_{n}^{*}(t)-s_{n}^{(q) *}(t)-s_{n}^{(j) *}(t)\right\} \leq-\epsilon \quad n \in \mathcal{N} \\
& \mathbb{E}\left\{s_{n}^{(q) *}(t)+\sum_{m \in \mathcal{H}_{n}} a_{m n}^{*}(t)-u_{n}^{*}(t)\right\} \leq-\epsilon \quad n \in \mathcal{N} \\
& \mathbb{E}\left\{s_{n}^{(j) *}(t)-\sum_{m \in \mathcal{H}_{n}} a_{n m}^{*}(t)\right\} \leq-\epsilon \quad n \in \mathcal{N} .
\end{aligned}
$$

Theorem 2: If Assumption 1 holds, then the time average total quality of information $\bar{y}_{0}$ is within $O(1 / V)$ of optimality under the quadratic policy, while all queue backlogs grows with $O(V)$.

Proof: See Section IV-A and IV-B. 


\section{A. Quality of Information vs. $V$}

The optimality of information quality versus parameter $V$ under the quadratic policy can be derived from (29) as

$$
\begin{aligned}
& \mathbb{E}\left\{L(\Theta(\tau+1))-L(\Theta(\tau))-V y_{0}(\tau) \mid \Theta(\tau)\right\} \\
& \leq \sum_{n \in \mathcal{N}}\left\{K_{n}(\tau) \mathbb{E}\left\{d_{n}^{*}(\tau)-s_{n}^{(q) *}(\tau)-s_{n}^{(j) *}(\tau) \mid \Theta(\tau)\right\}\right. \\
&+Q_{n}(\tau) \mathbb{E}\left\{s_{n}^{(q) *}(\tau)+\sum_{m \in \mathcal{H}_{n}} a_{m n}^{*}(\tau)-u_{n}^{*}(\tau) \mid \Theta(\tau)\right\} \\
&+J_{n}(\tau) \mathbb{E}\left\{s_{n}^{(j) *}(\tau)-\sum_{m \in \mathcal{H}_{n}} a_{n m}^{*}(\tau) \mid \Theta(\tau)\right\} \\
&\left.-V \mathbb{E}\left\{r_{n}^{*}(\tau) \mid \Theta(\tau)\right\}\right\}+A \\
& \leq A-V\left(y_{0}^{(\mathrm{opt})}+\delta\right)+\delta \sum_{n \in \mathcal{N}}\left[K_{n}(\tau)+Q_{n}(\tau)+J_{n}(\tau)\right]
\end{aligned}
$$

We have used the fact that an $\omega$-only policy does not depend on queues $\Theta(\tau)$. The last inequality is valid for every $\delta>0$. Therefore

$$
\mathbb{E}\left\{L\left(\Theta(\tau+1)-L(\Theta(\tau))-V y_{0}(\tau) \mid \Theta(\tau)\right\} \leq A-V y_{0}^{(\mathrm{opt})} .\right.
$$

Taking an expectation and summing from $\tau=0$ to $t-1$ :

$$
\mathbb{E}\left\{L(\Theta(t))-L(\Theta(0))-V \sum_{\tau=0}^{t-1} y_{0}(\tau)\right\} \leq A t-V t y_{0}^{(\mathrm{opt})} .
$$

With rearrangement and $L(\Theta(t)) \geq 0$, it follows that

$$
\sum_{\tau=0}^{t-1} \mathbb{E}\left\{y_{0}(\tau)\right\} \geq-\frac{A t}{V}+t y_{0}^{(\mathrm{opt})}-\frac{L(\Theta(0))}{V} .
$$

Dividing by $t$ and taking limit as $t \rightarrow \infty$, the performance of the quadratic policy is lower bounded by

$$
\liminf _{t \rightarrow \infty} \frac{1}{t} \sum_{\tau=0}^{t-1} \mathbb{E}\left\{y_{0}(\tau)\right\} \geq-\frac{A}{V}+y_{0}^{(\mathrm{opt})} .
$$

This shows that the system can be pushed to the optimality $y_{0}^{(\mathrm{opt})}$ by increasing $V$ under the quadratic policy.

\section{B. Total Queue Backlog vs. V}

Now consider the existence of an $\omega$-only policy with Assumption 1 to the conditional expectation of the drift-pluspenalty (29) under the quadratic policy:

$$
\begin{aligned}
& \mathbb{E}\left\{L\left(\Theta(\tau+1)-L(\Theta(\tau))-V y_{0}(\tau) \mid \Theta(\tau)\right\}\right. \\
& \quad \leq A-V y_{0}^{(\epsilon)}-\epsilon \sum_{n \in \mathcal{N}}\left[K_{n}(\tau)+Q_{n}(\tau)+J_{n}(\tau)\right] .
\end{aligned}
$$

Taking expectation and summing from $\tau=0$ to $t-1$ :

$$
\begin{aligned}
& \mathbb{E}\{L(\Theta(t))\}-\mathbb{E}\{L(\Theta(0))\}-V \sum_{\tau=0}^{t-1} \mathbb{E}\left\{y_{0}(\tau)\right\} \\
\leq & A t-V t y_{0}^{(\epsilon)}-\epsilon \sum_{\tau=0}^{t-1} \sum_{n \in \mathcal{N}} \mathbb{E}\left\{K_{n}(\tau)+Q_{n}(\tau)+J_{n}(\tau)\right\}
\end{aligned}
$$

With rearrangement and $L(\Theta(t)) \geq 0$, it follows that

$$
\begin{aligned}
& \sum_{\tau=0}^{t-1} \sum_{n \in \mathcal{N}} \mathbb{E}\left\{K_{n}(\tau)+Q_{n}(\tau)+J_{n}(\tau)\right\} \\
& \quad \leq \frac{A t}{\epsilon}+\frac{V}{\epsilon}\left(\sum_{\tau=0}^{t-1} \mathbb{E}\left\{y_{0}(\tau)\right\}-t y_{0}^{(\epsilon)}\right)+\frac{\mathbb{E}\{L(\Theta(0))\}}{\epsilon} \\
& \quad \leq \frac{A t}{\epsilon}+\frac{V}{\epsilon}\left(t y_{0}^{(\max )}-t y_{0}^{(\epsilon)}\right)+\frac{\mathbb{E}\{L(\Theta(0))\}}{\epsilon} .
\end{aligned}
$$

Dividing by $t$ and taking limit as $t \rightarrow \infty$, the time-averaged total queue backlog is bounded by

$$
\begin{aligned}
& \lim \sup _{t \rightarrow \infty} \frac{1}{t} \sum_{\tau=0}^{t-1} \sum_{n \in \mathcal{N}} \mathbb{E}\left\{K_{n}(\tau)+Q_{n}(\tau)+J_{n}(\tau)\right\} \\
& \leq \frac{A}{\epsilon}+\frac{V}{\epsilon}\left(y_{0}^{(\max )}-y_{0}^{(\epsilon)}\right) \text {. }
\end{aligned}
$$

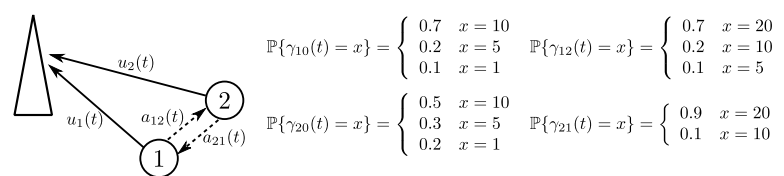

Fig. 2. Small network with independent channels with distributions shown

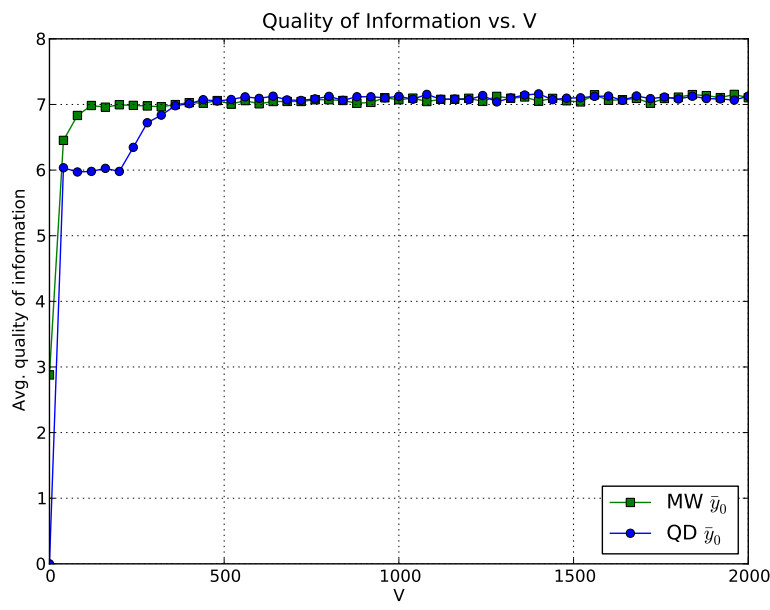

Fig. 3. Quality of Information versus $V$ under the quadratic (QD) and maxweight $(\mathrm{MW})$ policies

This shows that overall queue backlog tends to increase as $\mathrm{V}$ is increased.

The $V$ parameter in (30) and (31) affects the performance trade-off $[O(1 / V), O(V)]$ between quality of information and total queue backlog. These results are similar to those that can be derived under the max-weight algorithm. However, simulation in the next section shows significant reduction of queue backlog under the quadratic policy.

\section{Simulation Example}

Simulation under the proposed quadratic policy and the standard max-weight policy is performed over a small network in Fig. 2. The network contains two nodes, $\mathcal{N}=\{1,2\}$. Each node has the other as its neighbor, so $\mathcal{H}(1)=\{2\}$ and $\mathcal{H}(2)=\{1\}$. An event occurs in every slot with probability $\theta=0.3$. We assume all uplink and relay channels are orthogonal. The uplink channel distribution for node 1 is better than that of node 2 as in Fig. 2.

The constraints are $u_{n}(t) \in\left\{0, \ldots, \gamma_{n 0}(t)\right\}$ for $n \in \mathcal{N}$. Also, $a_{12}(t) \in\left\{0, \ldots, \gamma_{12}(t)\right\}$ and $a_{21}(t) \in\left\{0, \ldots, \gamma_{21}(t)\right\}$. Then set $s_{n}^{(q)(\max )}=s_{n}^{(j)(\max )}=30$. The feasible set of formats is $\mathcal{F}=\{0,1,2,3\}$ with constant options given by $\left(d_{n}^{(0)}, r_{n}^{(0)}\right)=(0,0),\left(d_{n}^{(1)}, r_{n}^{(1)}\right)=(100,20),\left(d_{n}^{(2)}, r_{n}^{(2)}\right)=$ $(50,15),\left(d_{n}^{(3)}, r_{n}^{(3)}\right)=(10,10)$ whenever there is an event.

The simulation is performed according to the algorithm in Section III-C. The time-averaged quality of information under the quadratic and max-weight policies are shown in Fig. 3. From the plot, the values of $\bar{y}_{0}$ under both policies converge to optimality following the $O(1 / V)$ performance bound.

Fig. 4abc reveals queue backlogs in the input, uplink, and relay queues under the quadratic and max-weight policies. At the same $V$, the quadratic policy reduces queue backlog 

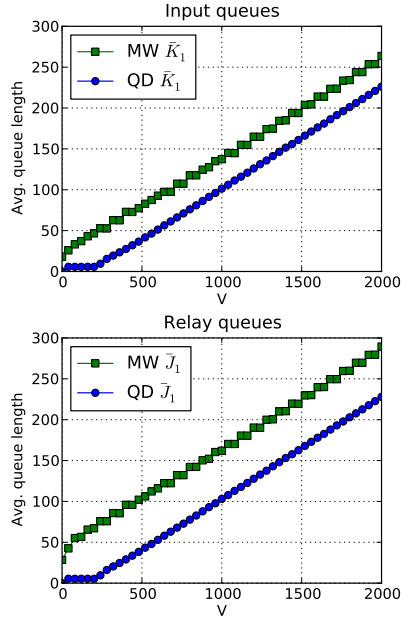
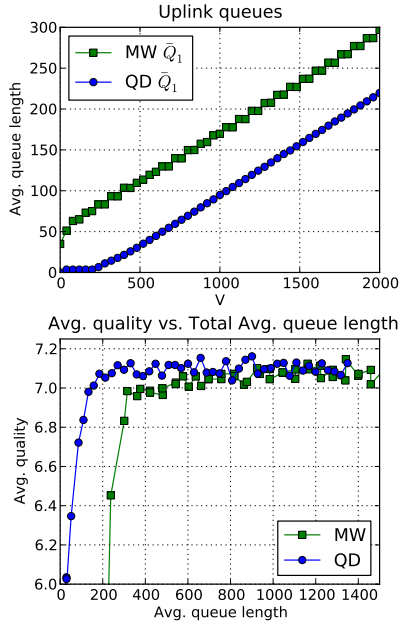

Fig. 4. Averaged backlog in queues versus $V$ and system quality versus backlog under the quadratic (QD) and max-weight (MW) policies

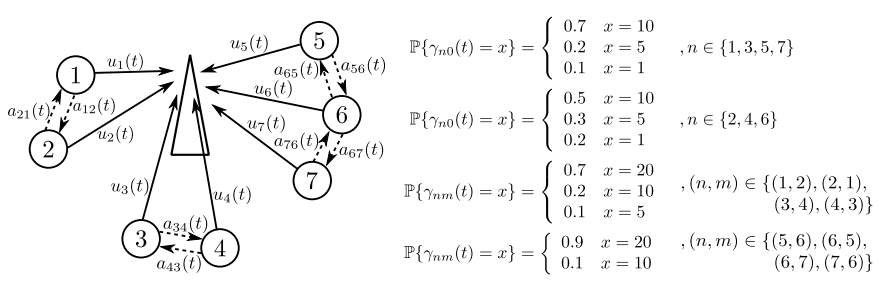

Fig. 5. Larger network with independent channels with distributions shown

by a significant constant compared to the cases under the max-weight policy. The plot also shows the growth of queue backlog with parameter $V$, which follows the $O(V)$ backlog bound.

Fig. $4 d$ shows that the quadratic policy can achieve near optimality with significantly smaller total system backlog compared to the case under the max-weight policy. This shows a significant advantage, which in turn affects memory size and packet delay.

Another larger network shown in Fig. 5 is simulated to observe convergence of the proposed algorithm. As in the small network scenario, the same probability of event occurrence $\theta=0.3$ is used. Channel distributions are configured in Fig. 5. For $V=800$, the time-averaged quality of information is 25.00 after $10^{5}$ time slots as shown in the upper plot of Fig. 6. The lower plot in Fig. 6 illustrates the early period of the simulation to illustrate convergence time.

\section{CONCLUSION}

We studied information quality maximization in a system with uplink and single-hop relay capability. From Lyapunov optimization theory, we proposed a novel quadratic policy having a separable property. In comparison with the maxweight policy, our policy leads to an algorithm that reduces queue backlog by a significant constant. This reduction also propagates and grows with the number of queues in the system. We simulated the algorithm to verify correctness and behavior of the new policy.
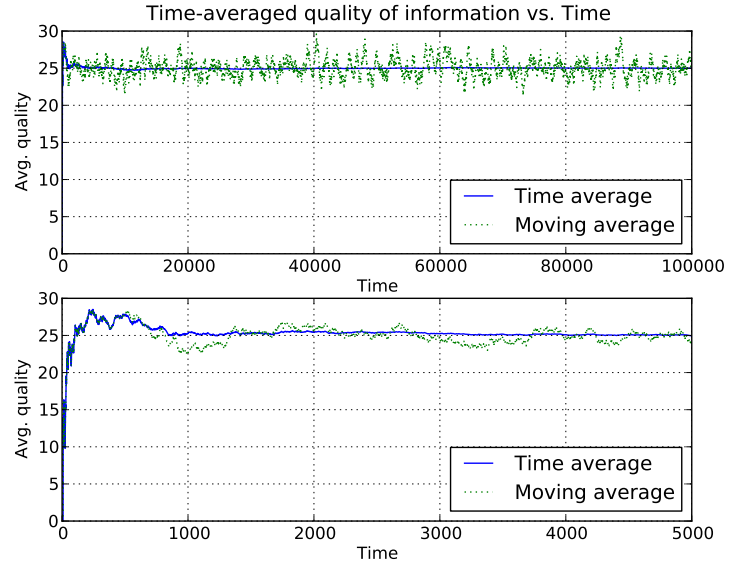

Fig. 6. Convergence of time-averaged quality of information

\section{ACKNOWLEDGMENT}

We would like to thank Ramesh Govindan for helpful discussions of this problem formulation.

\section{REFERENCES}

[1] E. Miluzzo, N. D. Lane, K. Fodor, R. Peterson, H. Lu, M. Musolesi, S. B Eisenman, X. Zheng, and A. T. Campbell, "Sensing meets mobile social networks: the design, implementation and evaluation of the cenceme application," ser. SenSys '08. ACM, 2008.

[2] S. Kang, J. Lee, H. Jang, Y. Lee, S. Park, and J. Song, "A scalable and energy-efficient context monitoring framework for mobile personal sensor networks," Mobile Computing, IEEE Transactions on, May. 2010.

[3] M. Mun, S. Reddy, K. Shilton, N. Yau, J. Burke, D. Estrin, M. Hansen, E. Howard, R. West, and P. Boda, "Peir, the personal environmental impact report, as a platform for participatory sensing systems research," ser. MobiSys '09. ACM, 2009.

[4] R. Y. Wang and D. M. Strong, "Beyond accuracy: what data quality means to data consumers," J. Manage. Inf. Syst., Mar. 1996.

[5] M. E. Johnson and K. C. Chang, "Quality of information for data fusion in net centric publish and subscribe architectures," in Int'l Conf. on Information Fusion, Jul. 2005.

[6] C. Bisdikian, L. M. Kaplan, M. B. Srivastava, D. J. Thornley, D. Verma, and R. I. Young, "Building principles for a quality of information specification for sensor information," Int'l Conf. on Information Fusion, 2009.

[7] C. H. Liu, C. Bisdikian, J. W. Branch, and K. K. Leung, "Qoiaware wireless sensor network management for dynamic multi-task operations," in Sensor Mesh and Ad Hoc Communications and Networks (SECON), Jul. 2010.

[8] A. Bar-Noy, G. Cirincione, R. Govindan, S. Krishnamurthy, T. F. LaPorta, P. Mohapatra, M. J. Neely, and A. Yener, "Quality-of-information aware networking for tactical military networks," in IEEE PERCOM Workshops, Mar. 2011.

[9] B. Liu, P. Terlecky, A. Bar-Noy, R. Govindan, and M. J. Neely, "Optimizing information credibility in social swarming applications," in IEEE INFOCOM, Apr. 2011.

[10] M. J. Neely, Stochastic Network Optimization with Application to Communication and Queueing Systems. Morgan \& Claypool, 2010.

[11] L. Georgiadis, M. J. Neely, and L. Tassiulas, "Resource allocation and cross-layer control in wireless networks," Foundations and Trends in Networking, 2006.

[12] L. Ying, S. Shakkottai, A. Reddy, and S. Liu, "On combining shortestpath and back-pressure routing over multihop wireless networks," IEEE/ACM Transactions on Networking, vol. vol. 19, no. 3, pp. 841854, Jun. 2011

[13] M. Chiang, S. H. Low, A. R. Calderbank, and J. C. Doyle, "Layering as optimization decomposition: A mathematical theory of network architectures," Proceedings of the IEEE, Jan. 2007.

[14] L. Tassiulas and A. Ephremides, "Stability properties of constrained queueing systems and scheduling policies for maximum throughput in multihop radio networks," Automatic Control, IEEE Transactions on, Dec. 1992. 